\title{
Implementing Quality Assurance in Public Health Facilities: The Malaysian Experience
}

\author{
Siti Haniza Mahmud, Nur Ezdiani Mohamed \\ Institute for Health Systems Research (Ministry of Health Malaysia), Shah Alam, Malaysia \\ Azman Abu Bakar \\ Medical Development Division (Ministry of Health Malaysia), Putrajaya, Malaysia
}

\begin{abstract}
The Quality Assurance Programme (QAP) in the public sector of the Ministry of Health (MOH) Malaysia had been in place for more than 25 years. It is an effort by the government to ensure that care provided is at par with the regional and the worldwide standards. The process toward attaining what was achieved today is neither an overnight nor an easy journey. Thus, this paper intends to share the implementation progress of QAP in the $\mathrm{MOH}$ government facilities for future benefits. To date, the MOH's champions in quality are well-recognized and had a strong governance structure that oversees the programme. Indicators were identified according to the needs of the activities in $\mathrm{MOH}$ and these measurements were used to measure the quality of the healthcare services in the government facilities. Sharing of best practices is done through local quality improvement journals and reports on the quality activities and research. Among the challenges faced is the process of gathering information. After 25 years, MOH is still collecting the information manually and hopefully, a better approach can be established.
\end{abstract}

Keywords: quality assurance (QA), government facilities, implementation

The health care industry in Malaysia adopted a dichotomous approach where the Ministry of Health $(\mathrm{MOH})$ Malaysia functioned as the main provider of the health care services in the country. The other providers are agencies within the public sector-Ministry of Education (teaching hospitals), Ministry of Defence (Army Hospital), and Ministry of Home Affairs along with statutory bodies and local authorities. The public sector is complemented by the private medical sector (hospitals and clinics) as well as the non-governmental organizations (Hamid et al., 1998). Till December 2013, there were 149 hospitals in the government sector in which 141 were governed by the $\mathrm{MOH}$. In the same year, there were 1,039 health clinics, 1,821 village clinics (klinik desa), 212 teams of mobile clinics, 13 teams of flying doctors, 52 oral health clinics, and 27 mobile oral health clinics. Apart from that, services in the urban poor areas were also provided by 2541 Malaysia Clinics and eight 1Malaysia Mobile Clinics which cover the remote areas far from a landed health clinic such as

Siti Haniza Mahmud, MD, MHP, Ph.D., Institute for Health Systems Research, Ministry of Health Malaysia, Malaysia; research field: health management. E-mail: sitihaniza.m@ihsr.gov.my.

Corresponding author: Nur Ezdiani Mohamed, MBBChBAO, Institute for Health Systems Research, Ministry of Health Malaysia; research field: health management. E-mail: nurezdiani.m@ihsr.gov.my.

Azman Abu Bakar, MBBS (UM), MPH (UM), Ph.D., Medical Development Division, Ministry of Health Malaysia, Malaysia; research fields: health management and health policy. E-mail: drazman@moh.gov.my. 
aboriginal and traditional village. On top of these, there were 214 registered private hospitals, 6,801 registered private medical clinics, and 1,686 registered private oral health clinics in the same year, 2013 (Bahagian Perancangan, 2014).

\section{The National Quality Assurance Programme in Health Care: The Beginning}

Apart from being a provider, $\mathrm{MOH}$ is also the regulator of the system. Most of the performances achieved thus far were due to the public sector. Thus, ensuring quality has always been one of our concerned. This is also in line with the policy and plan of the country as well as many other countries worldwide (Suleiman, Hamid, Hussein, Ming, \& Marikar, 2001). Since introducing the Quality Assurance Programme (QAP) on a national basis in 1985, we have aimed at continuously monitoring, evaluating, and improving the quality of health services in a planned and systematic manner (Hamid et al., 1998).

We started with Medical Services Division (for patient care) in 1985 through the use of selected outcome indicators to reflect the performance of our hospitals throughout the country. Since then, the programme has been extended to cover Health Services Division for promotive and preventive care (1990), Pharmaceutical Services Division (1990), Engineering Services Division (1992), Dental Services Division (1990), Laboratory Services (1992), and Training and Manpower Division (1996) (Suleiman et al., 2001).

The Quality Assurance Committee was set up at various levels of the Ministry of Health to ensure the smooth running of the programme as well as to define roles and responsibilities (see Figure 1). At the national level, the Health Ministry's National Steering Committee for the QAP is the "umbrella committee" for this programme. It is chaired by the Director-General of Health Malaysia and its members comprise various Programme Directors. Its role is to determine priorities, strategically plan for all QA (quality assurance) activities of all programmes, approve indicator introduction, refinement or deletion and to approve revision of standards.

At the divisional/programme, each division has its own Quality Assurance Programme Committee. The respective directors chair them. Their role is to develop and monitor QA activities, periodically review indicators and facilitate training activities. The Technical Committees for the various services advise each division's main committee in areas such as research and development as well as implementation and training.

However, at the state level, the State QA Committee is chaired by the various Directors of Health. On advice by the various Technical Sub-committees, the State QA committee members plan, coordinate and monitor QAP in their respective states as well as provide feedback to the $\mathrm{MOH}$. The states are thus more involved in implementation, problem-solving and capacity building.

Finally at the hospital/institutional level, the Hospital QA Committee addresses and is chaired by a Hospital QA Coordinator (who is a clinician). Members include the heads of the various services in the hospital. The QA coordinators are the prime movers of the QAP at this level.

This paper intends to share the implementation progress of the development of QAP in the MOH government facilities. 


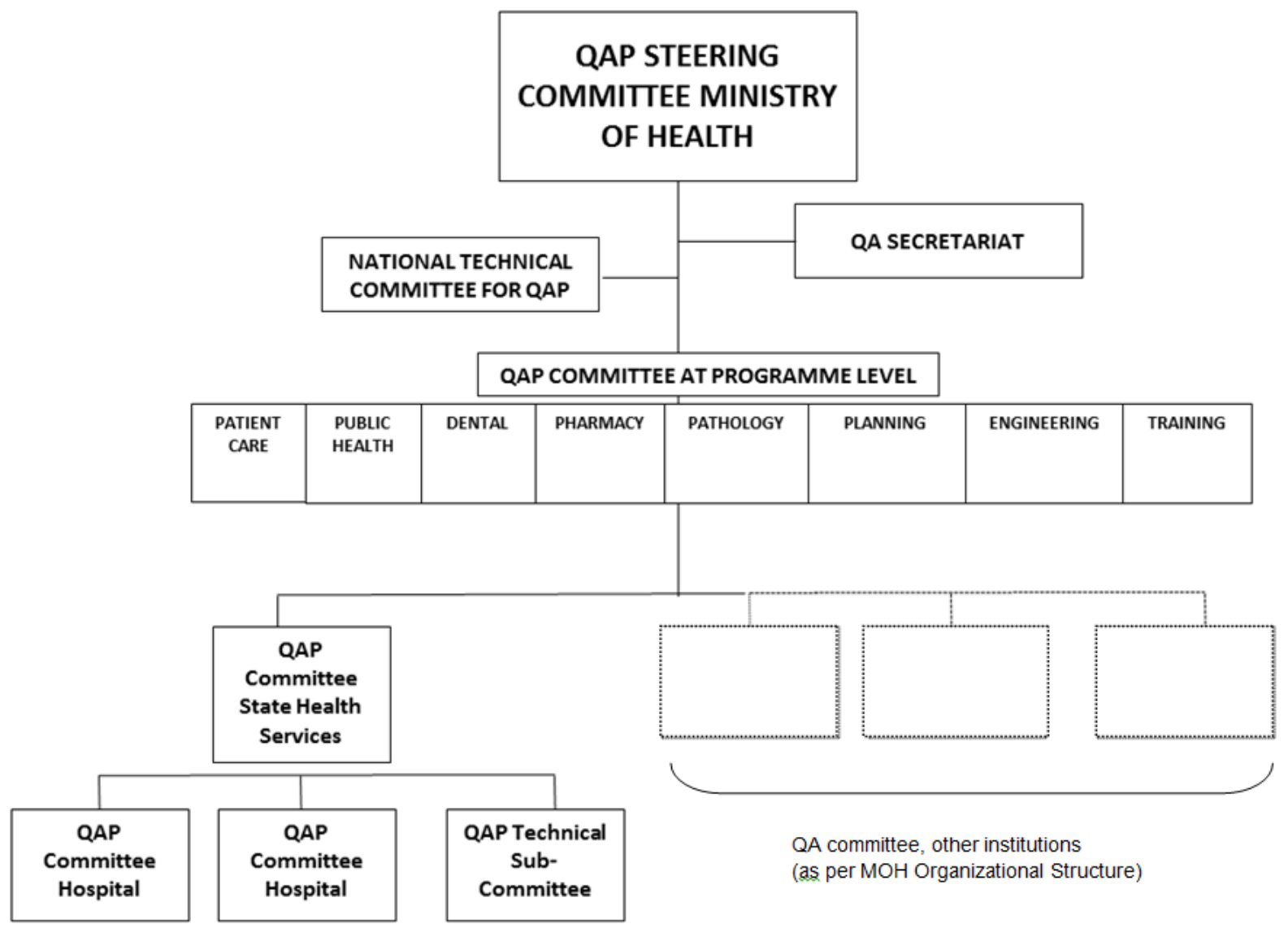

Figure 1. Organisational structure of the QAP in the MOH.

\section{Approach and Concept}

Commitment to QA is "one way in which commitment to quality is transformed from concept to reality" (Donabedian, 2003) and this commitment to quality has been reaffirmed by the MOH in its implementation of the National Quality Assurance Programme. In effect, the QAP formalised all the previous implicit and structural methods in ensuring quality and made the concern for quality explicit and system-wide. Quality has become everybody's business.

The goal of QAP is to ensure that, within the constraints of available resources, the patient, family, and the community obtain the "optimum achievable benefit" from the Health Ministry's services. This goal is consistent with the assertion that QA should be conducted within the aim of advancing the health and welfare of individuals and populations (Donabedian, 2003).

The authors developed the QAP using the Health Accounting Approach created by Donabedian and Williamson. A key concept in health accounting is ABNA (Achievable Benefit Not Achieved) which represents lost opportunity or potential not realised. The QAP aims at reducing the ABNA and to achieve this, it is imperative that resources at every level are utilised so that maximum benefit can be achieved.

In implementing the QAP and to achieve the specific objectives, a combination of two main strategies or approaches (which complement each other) was employed - the National Indicator Approach (NIA) and the Hospital Specific/District Specific Approach (HSA/DSA). In the "top-down" NIA, areas of concern which are common to most hospitals are identified. Indicators are then developed for these areas and data collected for 
each indicator are subsequently analysed. The performances are compared with set standards for each indicator. Reasons for "shortfalls in quality" are then analysed and remedial actions taken.

HSA/DSA on the other hand, adopted the "bottom-up" approach employed by local people solving their specific local problems. Hospitals and districts identify their own areas of concern and set their own standards for relevant indicators.

\section{Indicators Development to Measure Quality}

Indicators were developed for each area of concern in the major clinical disciplines such as medical, surgical, obstetric, and orthopedic in order to develop a standardized monitoring system. It was felt that it was not possible to adjust for case-mix, pre-admission, case-severity, chance occurrence, or other extraneous conditions outside the control of $\mathrm{MOH}$; the outcome indicators that were chosen could not be regarded as direct measures of quality. They were to be flags or signals that will indicate the potential problems which exist in the specific areas of concern.

In the beginning, the disciplines identified two problem areas that were considered important and relevant to most hospitals. These indicators were either rate-based or sentinel events. They were to serve as proxy indicators of care for a group of similar conditions or situations. The indicators also had to be acceptable to users to ensure ownership. With the passage of time and advancements in medical science, technology, and techniques, the suitability of these indicators were reviewed and reassessed.

We started off with the Patient Care Services in 1985 and to date, all the programmes have implemented QA on a staggered basis. Thus, the implementation of the QA programmes in the MOH is as shown in Table 1 . This national QAP is in various stages of implementation and maturity.

Table 1

Development in Implementation of QA Programmes

\begin{tabular}{lll}
\hline Programme & Year of implementation & $\begin{array}{l}\text { Number of indicators } \\
\text { (as of March 2010) }\end{array}$ \\
\hline Patient Care Services & 1995 & 58 \\
Public Health & 1990 & 16 \\
Pharmaceutical & 1990 & 16 \\
Laboratory & 1992 & 26 \\
Engineering & 1992 & 14 \\
Dental & 1992 & 5 \\
Training & 1996 & 5 \\
Planning \& Development & 1998 & 1 \\
\hline
\end{tabular}

The approach adopted in standard setting was to identify reasonable and attainable yardsticks. Standards were formulated through various approaches. One method used was reviewing historic data. Another approach was the use of national and international standards that were directly applied to the Malaysian setting or modified to suit the local situation as the incidence rate for post-operative infection of clean wound was $4 \%$, but the international standard is $2 \%$. Consensus among clinical experts was another method used in standard setting especially in HSA and DSA, where related problems which were being monitored were less complex than in the NIA. The experiences of these experts formed the baseline in determining arbitrary standards. However, if standards were unavailable from the literature, or when indicators were more complex, research projects will be carried out. 
These standards were used as a "flag", the measurement to indicate how well the authors are doing and able to make improvements. The achieved or non-achieved targets of the various government facilities will be compared across the facilities. Those facilities whose performances differed greatly from the majority are presumed to have a problem. Facilities that did not conform to the standards set will be required to carry out investigations using protocols drawn up for the indicator and these were known as quality improvement projects.

At present, the $\mathrm{MOH}$ has a total of 141 indicators at the national level and about 500 indicators at the hospital and district level in all the states. These indicators as well as the standards are continually monitored and refined to ensure that they are still appropriate, useful, and sensitive. Review and development of new indicators, while often a long and tedious process, has to be regularly undertaken in line with the changing trends in medical and health practices.

Table 2 displays a few examples of national indicators per programme.

Table 2

Examples of Selected National Indicators

\begin{tabular}{|c|c|c|}
\hline Name of indicator (standard set) & $\begin{array}{l}\text { Standard } \\
\text { (optimal target) }\end{array}$ & Formula \\
\hline Programme & \multicolumn{2}{|c|}{ Patient Care (General Medicine Service) } \\
\hline $\begin{array}{l}\text { ST Elevation Myocardial Infarct (STEMI) } \\
\text { Case Fatality Rate }\end{array}$ & $\leq 15 \%$ & $\begin{array}{l}\text { No. of patient admitted with STEMI \& who died from } \\
\text { STEMI } \times 100 \% \\
\text { Total no. of patient admitted with STEMI }\end{array}$ \\
\hline Programme & \multicolumn{2}{|c|}{ Patient Care (General Surgery) } \\
\hline Moderate Head Injury Case Fatality Rate & $\leq 10 \%$ & $\begin{array}{l}\text { No. of deaths due to moderate head injury } \times 100 \% \\
\text { No. of admissions with a diagnosis of moderate head } \\
\text { injury }\end{array}$ \\
\hline Programme & Oral Health & \\
\hline $\begin{array}{l}\text { Percentage of primary schoolchildren } \\
\text { maintaining orally fit status }\end{array}$ & $\geq 65 \%$ & $\begin{array}{l}\text { Total no. of NTR (No Treatment Required) primary } \\
\text { schoolchildren } \times 100 \% \\
\text { Total no. of new attendances of primary school } \\
\text { children }\end{array}$ \\
\hline Programme & Public Health & \\
\hline $\begin{array}{l}\text { Sputum conversion rate (SCR) for } \\
\text { Tuberculosis }\end{array}$ & $90 \%$ & $\begin{array}{l}\text { No. of smear +ve cases converted to }-\mathrm{ve} \text { after } 2 \\
\text { months of treatment } \times 100 \% \\
\text { No. of smear +ve cases at beginning of treatment }\end{array}$ \\
\hline Dengue outbreak control index (DOCI) & $\begin{array}{l}100 \% \text { of outbreaks must be } \\
\text { controlled within } 14 \text { days after } \\
\text { reporting of the } 2 \text { nd case }\end{array}$ & $\frac{\text { No. of dengue outbreaks controlled in a period }}{\text { Total no. of outbreaks in a period }} \times 100 \%$ \\
\hline
\end{tabular}

\section{Achievements}

Following its 25 years of establishment, Malaysian public healthcare delivery services have become among the best in the region (Suleiman et al., 2001). The Institute for Health Systems Research had been recognized as WHO (World Health Organization) Collaborating Center for Health Systems Research \& Quality Improvement since 1988. Malaysian QAP Champions were invited to provide consultation at other countries such as Brunei, Papua New Guinea, and Vietnam. Four modules in Quality Training had been developed and widely used across the Western Pacific Region (WPRO) countries.

Nationally, we have held conventions every two years starting from 2003. Findings from the QA research conducted were presented in the form of oral and poster presentations to share best practices among the public 
healthcare facilities. We also produce Quality Bulletin biannually which is disseminated throughout the country. Apart from that, at the state level, multiple echo training and state conventions were held and QI Journals or Bulletins were published. From 2004-2009, 173 personnel had been trained. Table 3 shows the details of the trainers.

Table 3

Categories of Personnel Trained

\begin{tabular}{lll}
\hline No. & Category & Total \\
\hline 1 & Clinical Specialists & 26 \\
2 & Medical officer & 95 \\
3 & Dietitian & 2 \\
4 & Pharmacists & 20 \\
5 & Dentists & 5 \\
6 & Scientists & 13 \\
7 & Administrative officers & 4 \\
8 & Engineers & 5 \\
9 & Nurses & 3 \\
& Total & 173 \\
\hline
\end{tabular}

\section{Challenges}

QAP has to face its own challenges along the way. Data collection for national indicators was done manually for reporting. The commitment and time needed to be sacrificed along with the day-to-day workload sometimes make the data given only at face value. Critical analysis was not done due to time constraint.

The mid-term evaluation report of the National Strategic Plan for Ministry of Health (2011-2015) reported that based on the Second Malaysia Burden of Disease and Injury, 2012, the burden of disease of the non-communicable disease (NCD) had increased from 69\% to 75\% in 2000 (Bahagian Perancangan, 2014). As NCD contributes to the chronic disease in Malaysia, this in turn increases the workload at the public side as most of the chronic patients seek care at the government facility.

As $\mathrm{MOH}$ is moving forward in providing better services for the nation, with the health sector reform coming on board, the challenge now lies in the effort of extending QAP to the private sector. Plan has to be developed and action has to be taken for them to incorporate quality assurance in their culture.

\section{Conclusions}

Implementation of QAP in the MOH government facilities is well-established. Following more than 25 years of implementation, QAP could be said is now at the mature stage of the growth graph. There are more challenges and issues need to be addressed for QAP to progress further and avoid the declining stage.

\section{References}

Donabedian, A. (2003). An introduction to quality assurance in health care. New York: Oxford University Press, Inc.

Hamid, M. A., Low, P., Abdullah, A. R., Yon, R., Morad, N., Hussein, R. H., ... Bakar, M. A. A. (1998). The strategic plan for quality in health. Kuala Lumpur: Percetakan Asni Sdn. Bhd.

Planning Division, MOH. (2014). MOH Strategic Plan 2011-2015: Mid-Term evaluation report. Putrajaya.

Suleiman A. B. (1995a). Experiences of Quality Assurance Programme in MOH, Malaysia. Proceedings from the World Health Organisation Pre-Congress Workshop on Methodologies in Quality Assurance. Canada. 
Suleiman, A. B. (1995b). Efficacy and efficiency in healthcare. Proceedings from the Combined Sceintific Meeting Chapter of Community and Occupational Physician. Academy of Medicine, Singapore.

Suleiman, A. B. (1995c). The approach of the MOH in further upgrading quality of health services. Proceedings from the World Bank Seminar on Implementing Population Programmes: Quality of Reproductive Care as the Way Forward. Kuching, Sarawak.

Suleiman, A. B., Hamid, M. A., Hussein, R., Ming, D. L., \& Marikar, M. A. K. (2001). Quality assurance in Malaysia. In A. F. AL-ASSAF (Ed.), Health care quality: An international perspective. New Delhi: World Health Organization. 